\title{
Tocilizumab treatment in juvenile idiopathic arthritis patients: A single center experience
}

\author{
Selcan Demir ${ }^{1}$, Hafize Emine Sönmez ${ }^{1}$, Elif Arslanoğlu-Aydın², Seza Özen ${ }^{1}$, Yelda Bilginer ${ }^{1}$ \\ ${ }^{1}$ Division of Rheumatology, ${ }^{2}$ Departments of Pediatrics, Hacettepe University Faculty of Medicine, Ankara, Turkey. \\ E-mail: yeldabilginer@yahoo.com \\ Received: 8th December 2017, Revised: 26th Januray 2018, 12th March 2018, Accepted: 27th July 2018
}

SUMMARY: Demir S, Sönmez HE, Arslanoğlu-Aydın E, Özen S, Bilginer Y. Tocilizumab treatment in juvenile idiopathic arthritis patients: A single center experience. Turk J Pediatr 2019; 61: 180-185.

Tocilizumab is a monoclonal antibody against interleukin- 6 that has recently emerged as an alternative treatment modality for juvenile idiopathic arthritis (JIA). In the present study, we aimed to discuss the clinical and laboratory findings and treatment response of JIA cases to tocilizumab therapy. This retrospective study included 20 JIA patients aged between 0-18 years who were followed up from 2014 to 2016 and received tocilizumab treatment in our clinic. Treatment response could be not evaluated in two patients since they developed anaphylactic reactions due to tocilizumab. Of the remaining 18 patients, seven of them $(38.9 \%)$ had polyarticular JIA, and eleven $(61.1 \%)$ had systemic JIA. Platelet counts, erythrocyte sedimentation rate and C-Reactive protein (CRP) levels, active joint counts, and Juvenile Arthritis Disease Activity Score 71 (JADAS71) were significantly decreased at the third month in both polyarticular and systemic JIA, while there were not any significant differences between the third and sixth months. All of the patients with polyarticular JIA had low disease activity at six months. Eight patients with systemic JIA had an inactive disease at six months, whereas the remaining three patients had high levels of CRP without presence of any clinical symptoms. Steroid treatment was terminated at the sixth month in all patients except for three patients who continued to receive 0.05-0.25 $\mathrm{mg} / \mathrm{kg}$ steroid treatment. Two patients developed thrombocytopenia, one patient developed macrophage activation syndrome, and one patient had elevated transaminases due to tocilizumab treatment. Previous studies have shown that tocilizumab treatment is well-tolerated, effective, and safe for use in JIA patients. In the present study, we also demonstrated the efficacy of tocilizumab treatment in JIA patients from our clinic.

Key words: juvenile idiopathic arthritis, tocilizumab, rheumatology.

Juvenile idiopathic arthritis (JIA) is one of the most common chronic rheumatic diseases in children. Diagnosis is made with the exclusion of other causes in the presence of arthritis lasting at least 6 weeks in children under 16 years of age. According to the International League of Associations for Rheumatology (ILAR) classification, JIA is divided into seven subgroups: systemic, rheumatoid factor (RF)positive polyarticular, RF-negative polyarticular, oligoarticular, psoriatic arthritis, enthesitisrelated arthritis (ERA), and unclassifiable arthritis. ${ }^{1}$

There are published guidelines for the use of biological therapies for patients with JIA. According to these guidelines, it is recommended to start biological treatment if the disease activity is moderate-high, despite three-months of methotrexate treatment in patients with JIA. ${ }^{2,3}$ Tocilizumab is a recombinant human monoclonal antibody developed against the interleukin-6 (IL-6) receptor ${ }^{4}$. In recent years, it has been shown to be effective and safe in biologic drug resistant JIA cases. 5,6

In this study, we aimed to present the clinical and laboratory findings and treatment responses of JIA patients who were treated with tocilizumab. 


\section{Materials and Methods}

The study group consists of JIA patients who were treated with tocilizumab and followed up between the years of 2014-2016 in the Department of Rheumatology of Hacettepe University Faculty of Medicine. All files were evaluated retrospectively, and all patients were anonymous. The study was approved by the ethical committee of Hacettepe University (GO17/921-19, 05, December, 2017).

Patients were classified as JIA according to ILAR classification criteria $^{1}$. The medical charts of JIA patients who had been treated with tocilizumab were reviewed retrospectively and demographic data, clinical manifestations, laboratory findings, and Juvenile Arthritis Disease Activity Score71 (JADAS71) at the initiation, and third and sixth months of treatment with tocilizumab were documented from patient charts. Also, drug-induced side effects were recorded. $M E F V$ variant analysis was also documented in medical files.

The JADAS71 score is a tool used to evaluate disease activity. It is based on four parameters: 1) physican's global assessment of disease activity on a $0-10$ visual analog scale (VAS); 2) patient's/parent's global disease assessment of well-being on a 0-10 VAS; 3) active joint numbers, evaluated in 71 joints; and 4) erythrocyte sedimentation rate (ESR) (normalized according to the 0-10 scale). It is calculated by using the formula: physician VAS + patient/parent VAS + active joint count+ ESR-20/10 (if the ESR $<20$, the score of 0 is given; if $>120$, the score of 10 is given $)^{7,8}$.

The response to tocilizumab was evaluated according to disease activity at the third and sixth months. The patients who had inactive or low disease activity were classified as responders to treatment. In patients with a diagnosis of polyarticular JIA, inactive disease was defined when the JADAS71 score was less than 1 and when the low disease activity was between 1.1-3.8. ${ }^{9}$ Due to unavailability of the standard threshold of JADAS71 for systemic JIA patients, inactive disease or low disease activity was defined as lack of fever, rash, serositis, splenomegaly, lymphadenopathy, and arthritis, as well as normal levels of ESR and CRP and decreasing JADAS71 scores for systemic JIA patients. ${ }^{10}$
All patients were screened for existing latent and active tuberculosis at initiation of the therapy and every 6 months. Tuberculin skin test (TST) and chest X-rays were performed. A positive TST result was accepted as a reaction of $10 \mathrm{~mm}$.

Tocilizumab treatment was given to patients with polyarticular JIA once every four weeks and to patients with systemic JIA once every two weeks, with the dose of $10 \mathrm{mg} / \mathrm{kg}$ to patients under $30 \mathrm{~kg}$, and $8 \mathrm{mg} / \mathrm{kg}$ to patients above $30 \mathrm{kgs}$.

\section{Statistical Analyses}

Statistical analyses were performed using SPSS software version 21. Normality of the distribution of variables was examined by visual (histogram and probability graphs) and analytical methods (Kolmogorov-Smirnov/ Shapiro-Wilk). Descriptive data were expressed as median (minimum-maximum) values. The Friedman test was used to compare the nonnormally distributed continuous parameters, and Wilcoxon test was performed to test significance of pairwise differences using Bonferroni correction to adjust for multiple comparisons.

\section{Results}

Twenty patients with the diagnosis of JIA were included in the study. However, two patients (one patient with polyarticular and one patient with systemic JIA) could not continue tocilizumab due to an anaphylactic reaction). Finally, 18 patients were evaluated. Of the 18 patients, seven $(38.9 \%)$ had polyarticular JIA, and eleven $(61.1 \%)$ had systemic JIA. The characteristics of patients at diagnosis are summarized in Table I. None of the patients had any concomitant disease history. One patient with polyarticular JIA had uveitis. All patients with systemic JIA had persistent course of disease. The median age (minimum-maximum) at tocilizumab initiation was $12(3-17)$ years in polyarticular JIA patients and 14 (3-17) years in systemic JIA patients. The median duration of the disease before tocilizumab initiation was $5(2-10.5)$ years in polyarticular JIA patients and $5.5(0.5-12)$ years in systemic JIA. Prior to the treatment with tocilizumab, all patients were receiving corticosteroids and methotrexate. Among polyarticular JIA 
Table I. The Characteristics of Juvenile Idiopathic Arthritis Patients (JIA) Treated with Tocilizumab.

\begin{tabular}{|c|c|c|}
\hline & Polyarticular JIA $(\mathrm{n}=7)^{\mathrm{a}}$ & Systemic JIA $(\mathrm{n}=11)^{\mathrm{a}}$ \\
\hline \multicolumn{3}{|l|}{ Patient characteristics } \\
\hline Gender, female, n (\%) & $2(28.5)$ & $4(36.3)$ \\
\hline Age at disease onset (years) & $4(1-13)$ & $5(1-13)$ \\
\hline Age at diagnosis (years) & $4(1-13)$ & $5.5(1.5-13)$ \\
\hline \multicolumn{3}{|l|}{ Laboratory findings } \\
\hline $\begin{array}{l}\text { White blood cell count, } \times 10^{3} / \mu \mathrm{L} \text { at } \\
\text { diagnosis }\end{array}$ & $13.9(6.7-34.5)$ & $13.1(6.8-42.1)$ \\
\hline Platelet count, $\mathrm{x} 10^{3} / \mu \mathrm{L}$ at diagnosis & $398(272-772)$ & $498(352-971)$ \\
\hline $\begin{array}{l}\text { Erythrocyte sedimentation rate, } \mathrm{mm} / \\
\text { hour }(0-20)^{*} \text { at diagnosis }\end{array}$ & $58(26-74)$ & $40(2-90)$ \\
\hline $\begin{array}{l}\text { C-reactive protein, } \mathrm{mg} / \mathrm{dl}(0-0.8) * \text { at } \\
\text { diagnosis }\end{array}$ & $10(1.3-16)$ & $7.5(1.5-17.4)$ \\
\hline Anti-nuclear antigen positivity, $\mathrm{n}(\%)$ & $0(0)$ & $0(0)$ \\
\hline Rheumatoid factor positivity, n (\%) & $3(42.8)$ & $0(0)$ \\
\hline $\begin{array}{l}\text { MEFV gene mutation } \\
\text { E148Q/E148Q } \\
\text { M694V/V726A } \\
\text { M680I/V726A } \\
\text { A744S/- }\end{array}$ & $\begin{array}{l}2(28.5) \\
1(14.2) \\
0(0) \\
0(0) \\
1(14.2)\end{array}$ & $\begin{array}{cc}3 & (27.2) \\
1 & (9) \\
1 & (9) \\
1 & (9) \\
0 & (0)\end{array}$ \\
\hline JADAS71 & $18(10-25)$ & $15(9-21)$ \\
\hline
\end{tabular}

*Normal range; ${ }^{a}$ Data expressed as median (minimum-maximum); JADAS71, juvenile arthritis activity score 71; MEFV, Mediterranean feVer

patients, all were receiving non-steroidal antiinflammatory drugs (NSAIDs) and etanercept (anti-tumor necrosis factor), and 6 (85.8\%) had used adalimumab (anti-tumor necrosis factor). Of the 11 systemic JIA patients, $8(72.7 \%)$ were treated with NSAIDs, $10(90.9 \%)$ used anakinra (anti-IL1), and $3(27.3 \%)$ used canakinumab (anti-IL1). Of the 18 patients, $12(66.7 \%)$ patients were treated with multiple biological agents. Patients had been on $0.5(0.2-1) \mathrm{mg} /$ $\mathrm{kg}$ steroid treatment when tocilizumab was initiated. $M E F V$ variant analysis was performed in all patients. Five patients (2 polyarticular JIA and 3 systemic JIA) carried MEFV mutations (Table I).

The clinical and laboratory findings of the 18 patients at initiation, and third and sixth months of tocilizumab treatment are summarized in Tables II and III. At the initiation of tocilizumab, polyarticular JIA patients had 9 (7-12) active joints and a JADAS71 score of 15 (7-24). Platelet counts, ESR and CRP levels, active joint counts, and JADAS71 were significantly decreased at the third month. However, there were no significant differences between the third and sixth months. Six of the patients $(85.8 \%)$ with polyarticular JIA had low disease activity at the third month, while one patient still had active disease. This patient reached low disease activity at the sixth month. Steroid therapy could be interrupted at the third month in all but one patient. However, steroid treatment was also stopped at the sixth month in this patient. Patients with systemic JIA had 5 (2-14) active joints and a JADAS71 score of $14(8-22)$ at the initiation of tocilizumab. In addition, four (36.4\%) systemic JIA patients had fever and hepatosplenomegaly. Acute phase reactants (ESR and CRP), active joint counts, and JADAS71 were significantly decreased at the third month. No significant differences between the third and sixth months were detected. At three months, eight patients $(72.7 \%)$ had an inactive disease, while the remaining three patients had high levels of CRP without presence of any clinical symptoms. Apart from these three patients, steroid treatment had been stopped. These three patients continued to use steroids at a dose of $0.05-0.25 \mathrm{mg} / \mathrm{kg} /$ day. Patients were treated with tocilizumab 
Table II. The Clinical and Laboratory Findings of Polyarticular JIA Patients at Initiation, $3^{\text {rd }}$ and $6^{\text {th }}$ Month of Tocilizumab (TOC) Treatment.

\begin{tabular}{|c|c|c|c|c|}
\hline Parameters & $\begin{array}{l}\text { At initiation of TOC } \\
\text { treatment }^{\mathrm{a}}\end{array}$ & $3^{\text {rd }}$ month $^{\mathrm{a}}$ & $6^{\text {th }}$ month $^{\mathrm{a}}$ & $P$ value \\
\hline White blood cell, $\times 10^{3} / \mu \mathrm{L}$ & $9.3(8.6-16.9)$ & $9.4(5.4-16.7)$ & $10.3(5.4-11.9)$ & 0.3 \\
\hline Platelet count, $\times 10^{3} / \mu \mathrm{L}$ & $451(319-870)$ & $260(169-154)$ & $350(153-526)$ & 0.004 \\
\hline $\begin{array}{l}\text { Erythrocyte sedimentation } \\
\text { rate, } \mathrm{mm} / \text { hour }(0-20)^{*}\end{array}$ & $49(16-73)$ & $2(2-15)$ & $5(2-22)$ & 0.003 \\
\hline $\begin{array}{l}\text { C-reactive protein, } \mathrm{mg} / \mathrm{dl} \\
(0-0.8)^{*}\end{array}$ & $7.2(4.1-12)$ & $0.5(0.1-4.6)$ & $0.6(0.1-0,8)$ & 0.001 \\
\hline Active joint count & $9(7-12)$ & $0(0-4))$ & $0(0-2)$ & 0.001 \\
\hline JADAS71 & $17(7-24)$ & $3(2-8)$ & $2.5(2-3,2)$ & 0.001 \\
\hline
\end{tabular}

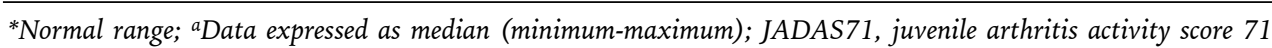

for a median duration of 15 (12-20) months.

During follow-up, thrombocytopenia in two patients, macrophage activation syndrome (MAS) in one patient, and the elevation of transaminase in one patient were observed. Tocilizumab treatment was interrupted for 2-4 weeks. These complications were not observed again when the medication was resumed after this short break. Anaphylaxis developed in two patients (in one patient at the second dose, and in another patient at the fifth dose). However, the treatment could not be continued because the families did not accept the desensitization protocol. In addition, uric acid levels and lipid profiles were evaluated among all patients at the first and six months. Uric acid levels and lipid profiles were all normal.

None of the patients had active pulmonary tuberculosis before initiation. Three patients had a TST above $10 \mathrm{~mm}$. Isoniazid (INH) prophylaxis was started in these three patients. INH was administered one month prior to starting tocilizumab and continued for nine months. None of the patients had activation of tuberculosis during follow-up.

\section{Discussion}

With the improvement in knowledge about the pathogenesis of JIA, cytokine-targeted treatment has become an agenda. Existing studies in JIA patients have shown increased levels of IL- 6 in synovial fluid and blood. ${ }^{11,12}$ Tocilizumab is the monoclonal antibody of the human IL-6 receptor, inhibiting IL- 6 binding its receptor by competitive binding and eliminating its proinflammatory effect. ${ }^{13}$ Tocilizumab has been approved for the treatment of systemic JIA and polyarticular JIA in children aged older than 2 years old who are refractory to conventional treatment ${ }^{14}$. In this study, we have summarized

Table III. The Clinical and Laboratory Findings of Systemic JIA Patients at Initiation, $3^{\text {rd }}$ and $6^{\text {th }}$ Month of Tocilizumab (TOC) Treatment.

\begin{tabular}{|c|c|c|c|c|}
\hline Parameters & $\begin{array}{l}\text { At initiation of TOC } \\
\text { treatment }^{\mathrm{a}}\end{array}$ & $3^{\text {rd }}$ month $^{\mathrm{a}}$ & $6^{\text {th }}$ month $^{\mathrm{a}}$ & $P$ value \\
\hline White blood cell, $\times 10^{3} / \mu \mathrm{L}$ & $9.7(6.8-23)$ & $6.8(5.4-13.8)$ & $6.9(5.3-14.3)$ & 0.009 \\
\hline Platelet count, $\times 10^{3} / \mu \mathrm{L}$ & $398(314-816)$ & $277(205-567)$ & $268(202-438)$ & $<0.001$ \\
\hline $\begin{array}{l}\text { Erythrocyte sedimentation } \\
\text { rate, } \mathrm{mm} / \text { hour }(0-20)^{*}\end{array}$ & $48(25-119)$ & $8(2-28)$ & $10(2-25)$ & 0.003 \\
\hline $\begin{array}{l}\text { C-reactive protein, mg/dl }(0- \\
0.8)^{*}\end{array}$ & $4.6(2.3-19)$ & $0.2(0.1-1.3)$ & $0.6(0.1-1.9)$ & $<0.001$ \\
\hline Active joint count & $5(2-14)$ & $0(0-2)$ & $0(0-2)$ & $<0.001$ \\
\hline JADAS71 & $15(7-24)$ & $4(1-9)$ & $3(2-4)$ & $<0.001$ \\
\hline
\end{tabular}

*Normal range; aData expressed as median (minimum-maximum); JADAS71, juvenile arthritis activity score 71 
our experiences of tocilizumab treatment in patients who had been followed-up in our clinic with the diagnosis of systemic JIA and polyarticular JIA.

The efficacy of tocilizumab in JIA patients was examined with two double-blind, randomized, placebo-controlled studies. The first study, which is entitled 'TENDER', was conducted by De Benedetti et al ${ }^{15}$. They evaluated 112 patients with a systemic JIA diagnosis who had persistent disease, with involvement of five or more active joints or two or more active joints along with fever, and were unresponsive to NSAIDs and corticosteroids. ${ }^{15}$ At the $12^{\text {th }}$ week, systemic findings like fever and rash, and laboratory findings such as anemia, thrombocytosis, and hyperferritinemia were significantly improved in patients receiving tocilizumab compared to the placebo group. At the $52^{\text {nd }}$ week, $80 \%$ of patients had at least $70 \%$ improvement, $59 \%$ of patients had $90 \%$ improvement in disease findings, and $32 \%$ of patients had achieved inactive disease. In addition, $48 \%$ of patients had no active arthritis, and oral glucocorticoid treatment was stopped in $52 \%$ of patients by the $52^{\text {nd }}$ week. ${ }^{15}$ The 'CHERISH' study, which included 188 patients, demonstrated the efficacy of tocilizumab treatment in patients with polyarticular JIA. ${ }^{16}$ Of patients treated with tocilizumab $64.6 \%$ had achieved American College of Rheumatology70 (ACR) response, and $45.1 \%$ of them had achieved ACR90 response at the end of study. ${ }^{16}$ In the present study, we demonstrated that the active joint numbers, acute phase reactants such as ESR and CRP, and JADAS71 scores were significantly decreased at the third month of treatment. Steroid treatment was stopped in all but three patients. Almost all of our patients had an inactive or low disease activity by the sixth month.

The higher frequency of mutations in the $M E F V$ gene among JIA patients has been previously reported. ${ }^{17,18}$ Mutations in the $M E F V$ gene may potentiate the inflammatory response in this inflammatory disease. Especially in countries where Familial Mediterranean Fever (FMF) is common, clinicians should be alert to this association. Thus, we performed MEFV gene variant analysis among all patients. Two patients with polyarticular JIA and three patients with systemic JIA carried MEFV mutations.
In addition to the effectiveness of tocilizumab treatment, side effects and safety have been evaluated by the conducted studies ${ }^{15}$, 16. Nasopharyngitis, headache, and upper respiratory tract infections were the most common adverse events according to previous studies. Cytopenia and elevated liver enzymes have been reported in patients receiving tocilizumab. Increased levels of transaminase in 21 patients were reported in the 'TENDER' study, and two patients had to discontinue treatment due to hypertransaminasemia. ${ }^{15}$ Furthermore, elevated alanine aminotransferase (ALT) levels in seven patients and thrombocytopenia in two patients were reported in the 'CHERISH' study. ${ }^{16}$ Thrombocytopenia in two patients and the elevation of transaminases in one patient were observed in our cohort. After the medications were interrupted, these laboratory abnormalities resolved spontaneously and did not recur when we re-administered the treatment. In the presented study, anaphylaxis was detected in two patients as a serious side effect.

Macrophage activation syndrome (MAS) is a serious, life-threatening complication of JIA. It can also occur during the use of biological therapy. Yokota et al. ${ }^{19}$ reported 23 MAS patients who were treated with tocilizumab, and after MAS symptoms recovered, tocilizumab was re-administered and MAS attack was not observed again in 18 patients. ${ }^{19}$ They suggested that there was no increased risk between tocilizumab treatment and MAS. ${ }^{19}$ In our study, MAS attack was observed in only one patient, and after the patient's clinical and laboratory findings were fully recovered, tocilizumab was re-introduced, and MAS attack was not observed again.

Turkey is a moderate risk country for tuberculosis, with an incidence rate of 21 per 100,000 inhabitants. BCG is still mandatory in the vaccination program, and latent tuberculosis is an important issue for our country. However, as previous studies have suggested a low risk of latent TB infection reactivation during tocilizumab therapy ${ }^{20,21}$, we routinely screen all patients before biologic drug initiation. Although the relatively small number of patient limits the study, in presented study we confirmed that tocilizumab therapy is a safe drug in terms of tuberculosis infection.

The major limitations of our study are the small 
number of patients and the single-centered and the retrospective characteristic of the study. In addition, all of the patients had been treated with another biologic agent before tocilizumab initiation.

Previous studies have demonstrated that the treatment of tocilizumab is a well-tolerated, effective, and safe medication in JIA patients. In our study, we have verified the efficacy of tocilizumab treatment in our systemic and polyarticular JIA patients who are resistant to treatment.

\section{REFERENCES}

1. Petty RE, Southwood TR, Manners P, et al; International League of Associations for Rheumatology. International League of Associations for Rheumatology classification of juvenile idiopathic arthritis: second revision, Edmonton, 2001. J Rheumatol 2004; 31: 390-392.

2. Ringold S, Weiss PF, Colbert RA, et al; Juvenile Idiopathic Arthritis Research Committee of the Children's Arthritis and Rheumatology Research Alliance (CARRA). Childhood Arthritis and Rheumatology Research Alliance consensus treatment plans for new-onset polyarticular juvenile idiopathic arthritis. Arthritis Care Res (Hoboken) 2014; 66: 1063-1072.

3. Beukelman T, Patkar NM, Saag KG, et al. 2011 American College of Rheumatology recommendations for the treatment of juvenile idiopathic arthritis: initiation and safety monitoring of therapeutic agents for the treatment of arthritis and systemic features. Arthritis Care Res (Hoboken) 2011; 63: 465-482.

4. Nishimoto N, Miyasaka N, Yamamoto K, Kawai S, Takeuchi T, Azuma J. Long-term safety and efficacy of tocilizumab, an anti-IL-6 receptor monoclonal antibody, in monotherapy, in patients with rheumatoid arthritis (the STREAM study): evidence of safety and efficacy in a 5-year extension study. Ann Rheum Dis 2009; 68: $1580-1584$.

5. Kostik MM, Dubko MF, Masalova VV, et al. Successful treatment with tocilizumab every 4 weeks of a low disease activity group who achieve a drug-free remission in patients with systemic-onset juvenile idiopathic arthritis. Pediatr Rheumatol Online J 2015; 13: 4.

6. Yokota $\mathrm{S}$, Itoh $\mathrm{Y}$, Morio $\mathrm{T}$, et al. Tocilizumab in systemic juvenile idiopathic arthritis in a real-world clinical setting: results from 1 year of postmarketing surveillance follow-up of 417 patients in Japan. Ann Rheum Dis 2016; 75: 1654-1660.

7. McErlane F, Beresford MW, Baildam EM, et al. Validity of a three-variable Juvenile Arthritis Disease Activity Score in children with new-onset juvenile idiopathic arthritis. Ann Rheum Dis 2013; 72: 1983-1988.

8. Consolaro A, Ruperto N, Bazso A, et al; Paediatric Rheumatology International Trials Organisation. Development and validation of a composite disease activity score for juvenile idiopathic arthritis. Arthritis Rheum 2009; 61: 658-666.
9. Demirkaya E, Consolaro A, Sonmez HE, Giancane G, Simsek D, Ravelli A. Current Research in Outcome Measures for Pediatric Rheumatic and Autoinflammatory Diseases. Curr Rheumatol Rep 2016; 18: 8.

10. Wallace CA, Giannini EH, Huang B, Itert L, Ruperto $\mathrm{N}$; Childhood Arthritis Rheumatology Research Alliance; Pediatric Rheumatology Collaborative Study Group; Paediatric Rheumatology International Trials Organisation. American College of Rheumatology provisional criteria for defining clinical inactive disease in select categories of juvenile idiopathic arthritis. Arthritis Care Res (Hoboken) 2011; 63: 929-936.

11. De Benedetti F, Pignatti P, Gerloni V, et al. Differences in synovial fluid cytokine levels between juvenile and adult rheumatoid arthritis. J Rheumatol 1997; 24: 1403-1409.

12. De Benedetti F, Martini A. Is systemic juvenile rheumatoid arthritis an interleukin 6 mediated disease? J Rheumatol 1998; 25: 203-207.

13. Nishimoto N, Kishimoto T. Inhibition of IL-6 for the treatment of inflammatory diseases. Curr Opin Pharmacol 2004; 4: 386-391.

14. Frampton JE. Tocilizumab: a review of its use in the treatment of juvenile idiopathic arthritis. Paediatr Drugs 2013; 15: 515-531.

15. De Benedetti F, Brunner HI, Ruperto N, et al; PRINTO; PRCSG. Randomized trial of tocilizumab in systemic juvenile idiopathic arthritis. N Engl J Med 2012; 367: 2385-2395.

16. Brunner HI, Ruperto N, Zuber Z, et al; Paediatric Rheumatology International Trials Organisation (PRINTO) and the Pediatric Rheumatology Collaborative Study Group (PRCSG).

16. Efficacy and safety of tocilizumab in patients with polyarticular-course juvenile idiopathic arthritis: results from a phase 3 , randomised, double-blind withdrawal trial. Ann Rheum Dis 2015; 74: 1110-1117.

18. Ayaz NA, Ozen S, Bilginer Y, et al. MEFV mutations in systemic onset juvenile idiopathic arthritis. Rheumatology (Oxford) 2009; 48: 23-25.

19. Ozcakar ZB, Cakar N, Uncu N, Celikel BA, Yalcinkaya F. Familial Mediterranean fever-associated diseases in children. QJM 2017; 110: 287-290.

20. Yokota S, Itoh Y, Morio T, Sumitomo N, Daimaru K, Minota S. Macrophage activation syndrome in patients with systemic juvenile idiopathic arthritis under treatment with tocilizumab. J Rheumatol 2015; 42: 712-722.

21. Lin CT, Huang WN, Hsieh CW, et al. Safety and effectiveness of tocilizumab in treating patients with rheumatoid arthritis - A three-year study in Taiwan. J Microbiol Immunol Infect 2019; 52: 141-150.

21. Ogata A, Mori M, Hashimoto S, et al. Minimal influence of tocilizumab on IFN-gamma synthesis by tuberculosis antigens. Mod Rheumatol 2010; 20: 130-133. 